\title{
Técnicas fisioterapêuticas para o manejo de tendinopatia patelar
}

\author{
Physiotherapeutic techniques for the management of patellar tendinopathy \\ Técnicas fisioterapéuticas para el tratamiento de la tendinopatía rotuliana
}

Recebido: 01/11/2021 | Revisado: 09/11/2021 | Aceito: 16/11/2021 | Publicado: 18/11/2021

André Luiz Cacau Martins Filho

ORCID: https://orcid.org/0000-0003-0394-5635 Centro Universitário da Amazônia, Brasil

E-mail: andreluizmartins84@gmail.com

Gabriel Santos de Jesus

ORCID: https://orcid.org/0000-0002-8083-5926 Centro Universitário da Amazônia, Brasil E-mail: gsantos1716@gmail.com

Otiniel Matos da Silva

ORCID: https://orcid.org/0000-0002-0256-9749 Centro Universitário da Amazônia, Brasil E-mail: otinielmatos@hotmail.com Wanderson Fernandes Silva

ORCID: https://orcid.org/0000-0001-9389-5795 Universidade do Estado do Pará, Brasil E-mail: wandersonfsm@outlook.com

\begin{abstract}
Resumo
Tendinopatia patelar é uma afecção relacionada com a sobrecarga do aparelho extensor do joelho. Essa lesão acomete o tendão patelar, causando dor à palpação e déficit funcional. O segmento mais acometido é a porção profunda e posterior do tendão patelar, adjacente ao pólo inferior da patela. Também é conhecida pelo nome de jumper's knee ou "joelho do saltador", por ser comum em atletas que praticam esporte de salto, podendo ser dividida em 4 graus de dor. A fisioterapia promove a melhora no quadro de dor, equilíbrio da musculatura, ganho de amplitude de movimento, possuindo um papel fundamental na recuperação da funcionalidade através de métodos terapêuticos, sendo realizada em conjunto com o uso de analgésicos e dispositivos de apoio. Os métodos de tratamento mais utilizados são a cinesioterapia, eletroterapia, alongamento, a bandagem patelar, além da crioterapia. O objetivo desse trabalho é discutir as técnicas fisioterapêuticas para o manejo de tendinopatia patelar. Metodologia: o artigo é uma revisão narrativa de literatura. Para a realização deste artigo foram realizadas filtragens nas plataformas da Google Scholar, Scielo e PubMed, os artigos selecionados foram todos relacionados ao tema proposto desde da parte etiologia da tendinopatia, passando pelas técnicas utilizadas no tratamento fisioterapêutico. Conclusão: As técnicas que mostraram maiores benefícios na fase aguda foram a cinesioterapia, principalmente pela execução dos exercícios excêntricos assim como as técnicas de alongamento que ajudar na flexibilidade do paciente e melhora da função motora, essa técnica além desses benefícios, também pode atuar na prevenção do desenvolvimento da tendinopatia. Nas fases crônicas da doença, a técnica que mostrou uma melhora no tratamento foi eletroterapia, já que ajuda na cicatrização e aumenta a força tênsil do tendão, para o controle da dor a técnica que mais se sobressaiu foi a crioterapia, uma vez que auxilia no alívio das dores localizadas mesmo ainda não havendo um consenso de sua aplicação adequada.
\end{abstract}

Palavras-chave: Fisioterapia; Déficit funcional; Tendinopatia.

\begin{abstract}
Patellar tendinopathy is a condition related to the overload of the knee extensor apparatus. This lesion affects the patella, causing pain on palpation and functional deficit. The most affected segment is the deep and posterior portion of the patellar tendon, adjacent to the inferior pole of the patella. It is also known by the name of jumper's knee or "jumper's knee", as it is common in athletes who practice jumping, and can be divided into 4 degrees of pain. Physiotherapy promotes improvement in pain, muscle balance, gain in range of motion, having a fundamental role in the recovery of functionality through therapeutic methods, being performed in conjunction with the use of analgesics and support devices. The most used treatment methods are kinesiotherapy, electrotherapy, stretching, patellar bandage, in addition to cryotherapy. The objective of this work is to discuss the physiotherapeutic techniques for the management of patellar tendinopathy. Methodology: the article is a narrative literature review. For the realization of this article, filtering was performed on Google Scholar, Scielo and PubMed platforms, the selected articles were all related to the proposed topic from the etiology of tendinopathy, through the techniques used in physical therapy treatment. Conclusion: The techniques that showed the greatest benefits in the acute phase were kinesiotherapy, mainly by performing eccentric exercises as well as stretching techniques that help in the patient's flexibility and improve motor function, this technique, in addition to these benefits, can also act in prevention of the development of tendinopathy. In the chronic phases of the disease, the technique that showed an improvement in the treatment was
\end{abstract}


electrotherapy, as it helps in healing and increases the tendon's tensile strength, for pain control the technique that most stood out was cryotherapy, as it helps in the relief of localized pain even though there is still no consensus on its adequate application.

Keywords: Physiotherapy; Functional deficit; Tendinopathy.

\section{Resumen}

La tendinopatía rotuliana es una condición relacionada con la sobrecarga del aparato extensor de la rodilla. Esta lesión afecta a la rótula, provocando dolor a la palpación y déficit funcional. El segmento más afectado es la porción profunda y posterior del tendón rotuliano, adyacente al polo inferior de la rótula. También se le conoce con el nombre de rodilla de saltador o "rodilla de saltador", ya que es común en los deportistas que practican el salto y se puede dividir en 4 grados de dolor. La fisioterapia promueve la mejora del dolor, el equilibrio muscular, la ganancia en el rango de movimiento, teniendo un papel fundamental en la recuperación de la funcionalidad a través de métodos terapéuticos, realizándose en conjunto con el uso de analgésicos y dispositivos de soporte. Los métodos de tratamiento más utilizados son la kinesioterapia, la electroterapia, el estiramiento, el vendaje rotuliano, además de la crioterapia. El objetivo de este trabajo es discutir las técnicas fisioterapéuticas para el manejo de la tendinopatía rotuliana. Metodología: el artículo es una revisión narrativa de la literatura. Para la realización de este artículo se realizó un filtrado en las plataformas Google Scholar, Scielo y PubMed, todos los artículos seleccionados estaban relacionados con el tema propuesto desde la etiología de la tendinopatía, a través de las técnicas utilizadas en el tratamiento de fisioterapia. Conclusión: Las técnicas que mostraron mayores beneficios en la fase aguda fueron la kinesioterapia, principalmente mediante la realización de ejercicios excéntricos así como técnicas de estiramiento que ayuden en la flexibilidad del paciente y mejoren la función motora, esta técnica, además de estos beneficios, también puede actuar en prevención del desarrollo de tendinopatía. En las fases crónicas de la enfermedad, la técnica que mostró una mejoría en el tratamiento fue la electroterapia, ya que ayuda en la cicatrización y aumenta la resistencia a la tracción del tendón, para el control del dolor la técnica que más destacó fue la crioterapia, ya que ayuda en el alivio. del dolor localizado aunque todavía no existe consenso sobre su adecuada aplicación.

Palabras clave: Fisioterapia; Déficit Funcional; Tendinopatía.

\section{Introdução}

A tendinopatia patelar é uma doença localizada na área conhecida como joelho; essa disfunção também é popularmente denominada de "joelho do saltador", já que em geral essa lesão se desenvolve em atletas de alta performance como corrida e saltos, já que requerem uma pressão subida no joelho. Pois essas atividades podem desenvolver micro traumas no tendão patelar, mas localizado na região chamada de polo inferior da patela (Andrade, 2003).

Os tendões são estruturas anatômicas conhecidas por serem abundantes em tecido conjuntivo que são responsáveis por conectar os músculos com as estruturas ósseas, o que possibilita a estabilidade e o movimento do corpo. No momento que os tendões se tornam enfraquecidos ou possuem alterações bioquímicas na estrutura pode ocorrer o desenvolvimento de uma disfunção nessa área, que é conhecida por tendinopatia patelar (Clark, 2013).

O tendão patelar é a estrutura que é responsável pela ligação distal do músculo chamado de quadríceps que possui origem na área no polo inferior da patela e se infiltra na parte superior da tuberosidade da estrutura denominada tíbia, que tem um tamanho que geralmente varia em torno de seis a oito centímetros (Basso, Johnson e Amis, 2001). Os tendões possuem estruturas de coloração esbranquiçadas e reluzentes, são conhecidos por serem resistentes e inextensíveis, sua composição é praticamente formada de tecido conjuntivo denso (Dangelo e Fattini, 2007).

Tradicionalmente, o termo tendinopatia se refere a uma doença não específica do tendão caracterizada por dor e função prejudicada devido ao processo de cicatrização inadequado do tendão (SpargolI et al., 2019). Essa doença é provocada por excesso de peso ou por movimento repetitivo que atinge um ou mais tendões, que pode desencadear sintomas como dor, inflamação, deformidades ósseas e muitas vezes, quando tornar-se crônica, além de acarretar inabilidade em determinados movimentos que requerem um esforço mais complexo do joelho (Clark, 2013).

Atualmente há duas hipóteses possíveis sobre o mecanismo lesional da tendinopatia patelar que pode se originar de maneira mecânica ou vascular. A teoria mecânica, relata sobre a ocorrência de microtraumatismos de episódios regularmente repetitivos, já a hipótese vascular afirma que a origem de tendinopatia seria que a lesão se desenvolve devido a redução da circulação sanguínea nas estruturas dos tendões (Cunha, Dias, Santos \& Lopes, 2012). 
Dessa maneira, nos dias atuais as duas hipóteses são aceitáveis e que podem existir simultaneamente para o desenvolvimento da tendinopatia patelar, sendo que qualquer uma delas de modo independente, entre as duas teorias possuem consequências semelhantes, o que torna as duas não menos verdadeiras, mesmo tendo aspectos diferentes entre as duas (Cunha, Dias, Santos \& Lopes, 2012).

Vários fatores de risco foram identificados, os quais podem ser divididos em fatores internos, como masculino ou aumento do índice de massa corporal, e fatores externos, como treinamento pesado ou habilidades de treinamento insuficientes, que parecem desempenhar um papel mais importante no desenvolvimento desta patologia, embora ainda não estejam bem identificados (Janssen, 2018; Rutland, 2010).

Para os quadros de tendinopatia patelar a comunidade médica indica fisioterapia e os exercícios físicos, que são técnicas que ajudam na diminuição de quadros de dor e também atuam na regeneração da função da estrutura em si. Atualmente os exercícios usados para fins terapêuticos têm sua efetividade comprovada por meio de revisões sistemáticas sobre a temática (Wasielewski e Kotsko, 2007).

\section{Metodologia}

O presente artigo é uma revisão narrativa de literatura, cujo o principal intuito é compilar informações a respeito de um determinado tema em um determinado espaço de tempo. Para o autor Polit, (2004) o propósito de uma revisão de literatura de pesquisa é agrupar os conhecimentos sobre um tema, corroborando nas ideias para o desenvolvimento de um trabalho totalmente novo, o que é crucial para as inovações médicas.

Dessa forma, os artigos que foram obtidos através de pesquisas nas plataformas de pesquisas Google Scholar, Pubmed e Scielo, já que a compilação de informações pelos meios eletrônicos é um grande avanço para área de pesquisa, o que a democratização do acesso é com esse auxílio a praticidade se manter os estudos atualizados (BrevidellI, 2008).

O método de inclusão seguida para o desenvolvimento do trabalho foram publicações dentro do período determinado de 2000 a 2021, assim como, estar dentro da temática específica de tendinopatia patelar. A amplitude da temática a ser pesquisada define o processo de coleta de material, ou seja, quanto mais específico for o objetivo da revisão mais rigoroso o processo de seleção deve ser quanto à inclusão da literatura (Broome, 2000).

$\mathrm{Na}$ etapa de exclusão dos artigos foi feito o delineamento do projeto onde foram excluídos 21 artigos no total, por não estarem se encaixando na temática requerida, assim como, por se encontrarem fora do período pré-determinado de 2000 a 2021, quando esses 2 fatores ocorrem é aceitável sua exclusão no desenvolvimento do projeto.

\section{Resultados e Discussão}

O presente artigo foi desenvolvido utilizando 43 artigos ao todo, sendo 14 artigos selecionados da plataforma da Google Scholar, 16 artigos coletados do banco de dados da Scielo (Scientific Electronic Library) e 13 retirados da base de dados da Pubmed. Todas as referências foram alocadas de forma coesa, fluída e objetiva, buscou-se que os arquivos selecionados abordassem de maneira significativa o tema, moldando o cerne coerente ao tema proposto.

Tendo em vista a necessidade de haver mais estudos a respeito da lesão do tendão patelar, especificamente a tendinopatia patelar, buscou-se elucidar de forma sucinta e coesa os procedimentos mais utilizados concernentes à área da Fisioterapia no tratamento desta lesão. Através da pesquisa de literaturas, os procedimentos mais utilizados foram a Cinesioterapia, Exercícios Excêntricos e Concêntricos, Eletroterapia, Alongamento, Mobilização Patelar, Bandagem Patelar e Crioterapia.

No decorrer da pesquisa, foram encontradas diversas literaturas no que tange às técnicas utilizadas no tratamento da tendinopatia patelar. De acordo com o material colhido, o tratamento da lesão do tendão patelar tem se mantido um desafio. 
Apesar de ser uma lesão relativamente comum, o conhecimento a respeito da eficácia dos tratamentos disponíveis ainda não é suficiente para postular um tratamento generalizado para todos os pacientes (Albuquerque, 2018).

De acordo com Pinto (2020), amplitude de movimento de dorsiflexão do tornozelo e redução da flexibilidade dos isquiotibiais, frequência de salto, melhor desempenho de salto reverso e saltos de aterrissagem mais difíceis (aterrissagens duras) foram identificados como fatores de risco para tendinopatia patelar. Como fatores relacionados, foram determinadas a diminuição da força muscular dos extensores, abdutores e rotadores externos do quadril, hiperpronação subtalar e diferenças de tamanho entre os membros inferiores, associadas à tendinopatia patelar. Também foi determinado que os atletas afetados pela doença passaram por mudanças na forma como realizam atividades físicas (como saltos). Tendo em mente a natureza multifatorial da tendinopatia patelar, a consideração desses fatores da cadeia dinâmica pode ser importante para a recuperação e potencial prevenção dessa condição.

Vale ressaltar que para o tratamento de tendinopatia patelar são utilizadas técnicas conjuntas para que o mesmo seja mais efetivo. No entanto, de acordo com Santos (2015), a fisioterapia tem efeito satisfatório no tratamento da dor e da funcionalidade em pacientes com tendinopatia patelar, sendo os exercícios excêntricos a técnica que apresentou melhores respostas de tratamento, independentemente do paciente ser atleta ou não, revelando-se excelente em relação a outras manejos técnicos fisioterapêuticos. As demais técnicas foram abordadas neste estudo, apontando seu procedimento e eficácia.

\subsection{Cinesioterapia}

Atualmente a cinesioterapia é a técnica que usa bases de informações anatômicas, biomecânicas e até mesmo fisiológicas com a finalidade de possibilitar ao paciente uma ocupação que ajude na prevenção de patologias, e se a patologia já está diagnosticada, essa técnica irá atuar como tratamento e reabilitação. A indicação dessa técnica é deveras cautelosa já que precisa de avaliação de um profissional, para traçar objetivos para o próprio tratamento, também serão necessárias reavaliações para atualização junto com a progressão do paciente até que se atinja o objetivo requerido (Guimarães, 2003).

A atividade física dentro da técnica de Cinesioterapia para o tratamento da tendinopatia patelar pode ser classificada de duas maneiras: passivo e ativo. Onde a forma da técnica passiva, compreende mecanismos e os aspectos em que o paciente possui uma participação no tratamento de forma passiva; os exercícios da técnica é realizado manualmente por outro indivíduo ou pelo profissional fisioterapêutica ou com auxílio de aparelhagens especiais, que simulam os movimentos fisiológicos ou que desempenham manipulações de formas dissemelhante segmentos ou tecidos, com ajuda de várias metodologias (Guimarães, 2003).

\subsection{Exercício excêntricos}

Uma modalidade dentro da cinesioterapêutica é o exercício nomeado como excêntrico já que abrange a chamada contração excêntrica, ou seja, uma a aplicação de carga muscular que envolve uma atividade de força externa com uma maior tensão durante as sessões de alongamento físico antes ou não da atividade física localizada na área do músculo patelar ou em qualquer área do corpo onde a lesão se encontre (Albert, 2002).

Esses exercícios vêm sendo desenvolvidos com mais frequência como tratamento fisioterápico não somente em caso de atletas de alta performance, mas também nos pacientes usuais, podendo ser realizada em laboratórios experimentais, em academias de ginástica ou até mesmo em clínicas de fisioterapia convencionais, já que é uma atividade que não requer tantos aparatos específicos para sua execução (Clebis e Natall, 2001).

Atualmente é um dos métodos de terapia por exercício mais comumente usados em programas de reabilitação para diferentes grupos de pessoas, sendo muito útil para programas de treinamento de pacientes idosos com doenças articulares a 
atletas amadores ou de elite. Muitos dos protocolos usados hoje são baseados em pesquisas sobre exercícios excêntricos projetados para tratar tendinopatia, prevenir lesões ou ganhar flexibilidade (Mcardle, 2002).

De acordo com Woodley, Newsham-West e David Baxter (2006), o exercício excêntrico pode neutralizar a falha causada pela tendinopatia, formando fibras de colágeno transversais no tendão, promovendo assim sua remodelação. Grande parte dos estudos mostrou que o treinamento excêntrico pode ter um impacto positivo no procedimento terapêutico da tendinopatia, mas poucos estudos de alta qualidade apoiam sua eficácia clínica com outros tratamentos, e as recomendações para programas específicos ainda são limitadas (Woodley; Newsham-West; David baxter, 2006).

Para Visnes et. al. (2007) mostrou através de um projeto de revisão onde o mesmo recomenda técnicas de tratamento através de treinamento excêntrico por possuir um efeito positivo. Os estudos disponíveis apresentam que o programa de tratamento deve incluir uma placa de declínio e deve ser efetuada ciente que vai haver algum nível de desconforto que os atletas que desenvolvem a patologia são indicados a retirada da prática esportiva. Todavia, esses aspectos requerem mais estudos.

Johnsson e Alfredson (2005) apontaram que, em comparação com o uso de treinamento com exercícios centrípetos dolorosos, os atletas com tendinopatia patelar crônica reduziram significativamente a dor e a perda de função durante o treinamento em declive com o treinamento do quadríceps excêntrico doloroso. No estudo de Young et al (2005), também foi comprovado que o plano de agachamento no declive descendente foi mais eficaz no tratamento da dor e da função motora de atletas com tendinopatia patelar.

Segundo os autores Leme e Fujita (2009), um programa de tratamento voltado para exercícios excêntricos do quadríceps se mostrou eficaz, que é umas das técnicas utilizadas na reabilitação das tendinopatias patelares, já que possui efeito positivo em relação a patologia, principalmente se as atividades estão sendo realizadas com o auxílio da ferramenta chamada prancha de declínio e possui grande chance de acarretar desconforto no decorrer de pelo menos 12 semanas.

Os resultados do estudo de Cunha et al (2012), realizado com atletas diagnosticados com tendinopatia patelar, mostraram que o agachamento pode ser realizado em descida com ou sem dor para reduzir os quadros de dores e assim melhorar a função. O estudo de Purdam et al (2004), também mostraram resultados aproximados: a realização de exercícios excêntricos em uma ladeira descendente reduziu a dor e retornou ao nível de atividade anterior após o treinamento.

\subsection{Exercício concêntricos}

Os exercícios concêntricos são aqueles movimentos de contração que encurtam os músculos. A maioria das atividades já desempenhou um papel na fase concêntrica. Segundo Gleeson (2003), no exercício excêntrico a contração muscular leva ao alongamento muscular, enquanto no exercício concêntrico, a contração muscular leva ao encurtamento muscular. Em relação ao movimento concêntrico, alguns autores determinaram que esse tipo de atividade promove aumento da força e hipertrofia muscular devido ao aumento da área de secção transversal e melhor ativação nervosa do grupo muscular em exercício. Porém, a eficácia dos exercícios não é compreendida na literatura (Lustosa, 2007). Embasado na literatura estudada, constatou-se que no caso de Tendinopatia Patelar, os exercícios excêntricos mostraram-se mais eficazes na recuperação e tratamento da lesão (Cannel et al, 2001).

\subsection{Eletroterapia}

A técnica chamada eletroterapia é efetuada como tratamento das tendinopatias patelares, na qual esse método possui meios de colaborar de maneira positiva com o acréscimo de resistência do colágeno das células de fibroblastos e consequentemente há uma maior resistência da estrutura do tendão. Os resultados apresentados nesses estudos são mais pertinentes, quando referentes na fase de cicatrização do tendão depois de um procedimento cirúrgico na área (Jardim, 2005). 
A técnica de ultrassom é muito utilizada na fase aguda pois ajuda a preservar o corpo dos efeitos térmicos e repetitivos nas lesões crônicas, esse método ajuda na parte da síntese de colágenos pelos fibroblastos, o que ajuda na cicatrização da lesão e ainda desencadeia um aumento da força tênsil no tendão do paciente. A técnica atualmente possui maior resultado a partir do funcionamento dos fibroblastos, na fase do reparo tecidual. No entanto, quando a patologia entra em estágio crônico, a técnica é mais utilizada, mas é frequentemente no estágio chamado de remodelação (Hebert, 2003).

O tratamento utilizando ondas de choque atualmente é considerado um método de grande sucesso para serem adotadas em casos de tendinopatias patelar quando em fase crônica (Chung, wiley.2002;). Esse modo de utilizar ondas de choque como forma de tratamento para as patologias de tendinopatias em fase crônica nos estudos apresentados recentemente, na qual esse distúrbio é caracterizado como dores recorrentes e degenerescência do tendão por um tempo estimado mínimo de seis meses (Wang et al, 2007).

Os resultados mostrados através de estudos foram muito oportunos para o tratamento da patologia de tendinopatias tanto na forma aguda quanto na forma crônica. Onde os autores chegaram à conclusão que o uso de eletricidade de maneira controlada, torna-se mais efetiva para se obter resultados positivos no tratamento mais conservador dos pacientes diagnosticados com tendinopatia patelar principalmente na forma crônica (Wang et al, 2007).

\subsection{Alongamento}

Todas as manobras terapêuticas desenvolvidas com o objetivo de melhorar a mobilidade de tecidos moles e a ADM são chamadas de alongamento. Durante o alongamento, a forma como o quadril está posicionado interfere na flexibilidade dos flexores e extensores do joelho. A avaliação da flexão dos músculos posteriores da coxa e do reto femoral deve ser realizada à parte (Kisner, 2005).

O alongamento é benéfico para aumento da flexibilidade e melhora da função motora. É necessário para a prevenção e reabilitação de lesões. O mais comumente utilizado é o alongamento estático que é aplicado com segurança de acordo com a tolerância do paciente. Os exercícios de flexibilidade para membros inferiores desempenham um papel importante que minimiza a pressão na patela (Peccin; Chamlian, 2005). Os programas de reabilitação que utilizam as técnicas de exercícios de alongamento e fortalecimento muscular variam muito. A maioria dos autores defende a recuperação total, incluindo fortalecimento, flexibilidade, padrões de movimento, propriocepção, resistência e progresso gradual.

\subsection{Mobilização patelar}

Pode ser realizado por deslizamento lateral-interno, e o alongamento da estrutura lateral pode ser realizado passivamente pelo fisioterapeuta por meio da técnica de movimentação da cinta de suporte lateral, que pode ser realizada pelo próprio paciente. A técnica envolve colocar a área tenar da mão na borda medial da patela e aplicar pressão posterior na borda medial. Isso fará com que as bordas laterais se movam para frente, esticando o retináculo (Hebert, 2003).

Na mobilização da patela, com a desconexão medial, o paciente deve estar em decúbito lateral, com uma das mãos estabilizando o côndilo femoral abaixo do fêmur e a base da outra deslizando a patela medialmente. Quando a parte interna da patela está inclinada, o paciente deve deitar-se de costas e colocar a protuberância tênar na superfície interna da patela. A força de retorno à direita faz com que a patela se incline medialmente (Kisner, 2005).

Os autores Hall \& Brody (2001), relatam que a remobilização possui o poder de auxiliar na recomposição das características mecânicas e organizações teciduais dos ligamentos, no entanto o período essencial para essa regeneração seja realizado de maneira benéfica para o paciente ainda não foi determinado de maneira exata. Segundo relatos do autor Davisson et al, (2002) as forças de cisalhamento e compressivas são métodos que são desenvolvidas na superfície articular do indivíduo na qual é desencadeada pelo movimento que provoca um aumento na síntese do colágeno assim como de proteoglicanos. 
A técnica funciona auxiliando na luta contra as consequências negativas decorrentes da imobilização e além de desencadear uma carga que irá agir sobre os tecidos moles o que torna à mobilização importante no retorno da reparação tecidual. O método tem o potencial ajudar a manter a área da lesão mais exequível já que a parte mecânica são fundamentais no período do reparo-regeneração, uma vez que elas ajudam na parte de orientação das fibras colagênicas que foram recentemente produzidas ao longo das linhas de estresse da lesão (Hall \& Brody, 2001).

Para o autor Sorrenti (2006), o fundamento principal é a preocupação com a mobilização precoce possui um risco de ocasionar uma nova lesão ou abertura da lesão que está sendo tratada da área do tendão, mas acaba sendo reduzida em indivíduos que são orientados a prosseguirem com protocolo de tratamento mobilização precoce. Os resultados de vários estudos fortalecem o argumento de que o método de mobilização precoce na reabilitação após a reparação cirúrgica é eficaz e ajuda na recuperação do tendão patelar.

\subsection{Bandagem patelar}

É usado para reajustar a patela e alongá-la durante o treinamento de baixa intensidade para mantê-la alinhada. O uso de materiais viscoelásticos e o uso de bandagens nos tendões são de valor controverso e têm o objetivo de aliviar a dor causada pela divergência de forças. O uso prolongado enfraquece os tendões e agrava a lesão, obrigando os atletas a usarem curativos por muito tempo. Só é utilizado por atletas profissionais, em caso de extrema necessidade de competição, é muito difícil, lento e trabalhoso retirar o curativo. Deve ser lembrado que após qualquer técnica passiva, algum movimento ativo das articulações é necessário (Herbert, 2003).

Recomenda-se o uso de bandagens esportivas ou bandagens duras para tratar lesões agudas e prevenir lesões. Considerando que é utilizado imediatamente antes da atividade e retirado imediatamente após a atividade, irá produzir excelente suporte articular e limitar os movimentos articulares anormais ou excessivos, pois sua composição contém grande quantidade de látex, podendo irritar a pele, aumentar a transpiração e a opressão (Branco et al, 2016).

Segundo Ribeiro et al (2010), o enfaixamento funcional principalmente para tendinopatia patelar é um tratamento considerado conservador que possui diversas finalidades, principalmente para ganhar o controle neuromuscular (a atividade do quadríceps, o início da atividade do músculo oblíquo medial relacionado ao músculo femoral lateral e a movimentação do joelho durante a atividade).

Para o autor Salgado (2002), relatou que os curativos funcionais são uma ferramenta indispensável na recuperação de atletas, que podem promover a reabilitação rápida dos atletas e evitar que os atletas percam a função, pois promove a recuperação precoce das atividades, protegendo a parte machucada de possíveis lesões futuras. (DERASARI et al, 2010). De acordo com as pesquisas realizadas, a eficácia dos curativos funcionais no alívio da dor e na manutenção da amplitude de movimento pode ser verificada em toda a literatura pesquisada. De acordo com a pesquisa de Derasari (2010), enfatizou-se a importância da biomecânica do joelho nas soluções de alívio e dor.

\subsection{Crioterapia}

A crioterapia ou terapia fria é a aplicação de qualquer substância no corpo, fazendo com que a temperatura do corpo e dos tecidos diminua (Ferreira; Fernandes, 2012; Branco et al, 2005). O calor é expelido do corpo e absorvido pelo frio, e respostas locais e sistêmicas são obtidas, as quais estão relacionadas à temperatura, duração do tratamento e área de tratamento (Starkey, 2001; Freitas; Luzardo, 2013).

Considerando o efeito analgésico do gelo e o efeito de redução da taxa metabólica, além de reduzir o extravasamento de sangue e proteína em novos capilares, recomenda-se o uso de gelo nas lesões tendíneas do tendão patelar. Seu papel 
também se estende para desacelerar a inflamação, promover leucopenia e o efeito da histamina nas membranas dos vasos sanguíneos (Jardim, 2005).

Apesar de seus benefícios, até o momento, não há consenso sobre sua forma de uso, ou seja, a temperatura, o tempo e a quantidade ideais de uso. O gelo é um tratamento acessível e popular entre os médicos, portanto, seu uso deve ser apoiado por evidências científicas mais consistentes (Jardim, 2005). No entanto, ainda não há consenso sobre a aplicação exata da crioterapia.

\section{Considerações Finais}

Através dos resultados da pesquisa podemos analisar que as técnicas são utilizadas de acordo com o estado que a tendinopatia patelar se encontra, através dessa análise o fisioterapeuta repassa o tratamento ideal para a recuperação do indivíduo. As técnicas que mostraram maiores benefícios na fase aguda foram a cinesioterapia, principalmente pela execução dos exercícios excêntricos assim como as técnicas de alongamento que ajudar na flexibilidade do paciente e melhora da função motora, essa técnica além desses benefícios, também pode atuar na prevenção do desenvolvimento da tendinopatia. Nas fases crônicas da doença, a técnica que mostrou uma melhora no tratamento foi eletroterapia, já que ajuda na cicatrização e aumenta a força tênsil do tendão, para o controle da dor a técnica que mais se sobressaiu foi a crioterapia, uma vez a mesma auxilia no alívio das dores localizadas mesmo ainda não havendo um consenso de sua aplicação adequada. As outras técnicas, apresentaram benefícios em diferentes graus uns maiores que outros por esse fator, ainda há necessidade de mais pesquisas a fim de aperfeiçoar as técnicas, em especial a técnica de crioterapia, pois já se sabe dos seus benefícios, mas não do seu método de aplicação correto.

\section{Referências}

Andrade, A. P. E., Nogueira, S. R. S., \& Heluy, G. D. (2012). Tendinite patelar: resultado do tratamento cirúrgico.

Albert, M. (2002). Treinamento excêntrico em esportes e reabilitação (1 $\left.{ }^{\mathrm{a}} \mathrm{ed}.\right)$. Manole.

Albuquerque, FMP (2018). Tendinopatia do Tendão Rotuliano [Dissertação de Doutorado, Repositório Digital da UBI]. http://hdl.handle.net/10400.6/8399.

Basso, O., Johnson, D., \& Amis, A. (2001). The anatomyofthepatellartendon. KneeSurg , (9), 2-5.

Bahr R, Fossan B, Løken S, Engebretsen L. Surgical treatment compared with eccentric training for patellar tendinopathy (Jumper's Knee). A randomized, controlled trial. the Journal of Bone \& Joint Surgery. 2006;88(8):1689-98.

Branco, G. (2016). Princípios em técnicas de Taping.Disponível em: Acesso em 18 Set. 2016. https://docplayer.com.br/31538628-Principios-em-tecnicas-detaping.html

Branco, P. S., Martelo, D., Constantino, H., Lopes, M., José, R., Tomás, R., \& Carvalho, T. (2005). Tema de reabilitação agentes físicos: crioterapia, termoterapia, diatermia (1st ed.). Medesign.

Brevidell, M., \& Domenico, E. (2008). Trabalho de conclusão de curso: guia prático para docentes e alunos da área da saúde (2a ed.). Iátria.

Broome, M. (2000). Revisões integrativas da literatura para o desenvolvimento de conceitos. In: Rodgers BL, Knafl KA, editores. Desenvolvimento de conceitos em enfermagem: fundamentos, técnicas e aplicações. ; 2000. p.231-50. Filadélfia (EUA): WB Saunders Company , 231-50.

Clark, B. (2013). Tendinopatia é causada pelo excesso de uso dos tendões do pé e tornozelo. Rev. Fisio Personalizada .

Cannell, L., Taunton, J., Clement, D., Smith, C., \& Khan, K. (2001). Um ensaio clínico randomizado da eficácia do agachamento ou exercícios de extensão / flexão de perna para tratar joelho de saltador com diagnóstico clínico em atletas: estudo piloto. British Journal of Sports Medicine , 35 (1), $60-4$.

Cunha, R. A. D., Dias, A. N., Santos, M. B., \& Lopes, A. D. (2012). Estudo comparativo de dois protocolos de exercícios excêntricos sobre a dor e a função do joelho em atletas com tendinopatia patelar: estudo controlado e aleatorizado; Comparative study of two protocols of eccentric exercise on knee pain and function in athletes with patellar tendinopathy: randomized controlled study. Revista Brasileira de Medicina do Esporte.18(3), 167-170.

Campbell, R., Grainger, A. (2001). Current concepts in imaging of tendinopathy. Clinical Radiology l, 56(4), $253-67$.

CLEBIS, Naianne, NATALL, \& Maria. (2001). Lesões musculares provocadas por exercícios excêntricos. Revista Brasileira de Ciência e Movimento , 9, (4), 47-53.

Chung, B., \& Wiley, J. (2002). Extracorporeal shockwave therapy. Sports Medicine, 32(13), 851-65. 
Dangelo, JG, \& Fattini, CA (2007.). Anatomia humana sistêmica e segmentar (3 ${ }^{\text {a }}$ ed.). São Paulo, Rio de Janeiro, Belo Horizonte: Atheneu.

Derasari, A., Brindle, T., Alter, K., \& Sheehan, F. (2010). McConnell Taping desloca a patela inferiormente em pacientes com dor patelofemoral: um estudo de ressonância magnética dinâmica. Fisioterapia, 90 (3), 411-419.

Santos, F. T., \& Piazza, L. (2015). Evidências científicas no tratamento fisioterapêutico da tendinopatia patelar: uma revisão sistemática da literatura. ConScientiae Saúde, 14(3), 489-496.

Ferreira, A. A. S., \& Fernandes, D. S. S. L. (2012). Influência da Crioterapia e do calor ultrassônico na paralisia cerebral: relato de caso. Revista Neurociencia, 20(4), 552-559.

Freitas, C., \& Luzardo, R. (2013.). Crioterapia: efeitos sobre as lesões musculares. Revista Episteme Transversalis, 4(1), 1-6.

Guimarães, S. L; Cruz, C. (2003.). Exercícios terapêuticos: a cinesioterapia como importante recurso da fisioterapia. Lato \& Sensu, 4(1), 3-5.

Gleeson, N., Eston, R., Marginson, V., McHugh, M., \& Bird, S. (2003). Effects of prior concentric training on eccentric exercise induced muscle damage. British journal of sports medicine, 37(2), 119-125.

Hall, M., \& Brody, T. (2001). Exercício Terapêutico na Busca da Função. Editora Guanabara Koogan , 1 (1), $172-174$.

Janssen I, van der Worp H, Hensing S, Zwerver J. Investigating Achilles and patellar tendinopathy prevalence in elite athletics. Res Sport Med [Internet]. 2018;26(1):1-12. Available from: https://doi.org/10.1080/15438627.2017.1393748

Jardim, M. (2005). Tendinopatia Patelar. Arq. Essfisioonline, voll, $N^{\circ} 4$, setembro / 2005, 1 (4), 1-102.

Jonsson, P., \& Alfredson, H. (2005). Resultados superiores com treinamento excêntrico em comparação com o quadríceps concêntrico em pacientes com joelho de saltador: um estudo prospectivo randomizado. British Journal of Sports Medicinev. 39, pág. 847-50, 2005, 39 (11), 847-50.

Leme, G. P. C., \& Fujita, A. P. (2009). Efetividade do treinamento muscular excêntrico no tratamento da tendinopatia patelar. Ensaios e Ciência: C. Biológicas, Agrárias e da Saúde, 13(2), 111-124.

Lustosa, L. P. (2007). Benefícios dos exercícios excêntricos e concêntricos dentro do programa de fortalecimento muscular. Fisioterapia Brasi, 8(4), $283-287$.

Mcardle, Ferrer-CAJA, Hamagami, \& Woodcock. (2002). Análises Estruturais Comparativas Longitudinais do Crescimento e Declínio de Múltiplas Habilidades Intelectuais ao Longo da Vida. Developmental Psychology, 38 (1), 115-142.

Panni, A., Biedert, R., Maffulli, N., Tartarone, M., \& Romanin, E. (2002). Lesões por uso excessivo do mecanismo extensor em atletas. Clinical Journal of Sport Medicine, 21 (3), 483-98.

Peccin, MS, Chamlian, TR, Princípios da reabilitação femoropatelar. In :Cohen, M, M., \& Abdalla, RJ (2005). Lesões nos esportes: diagnóstico, prevenção e tratamento. Rio de Janeiro: Revinter , 575-578.

Pinto, N. R. (2020). Fatores de risco para tendinopatia patelar relacionados à cadeia cinética: estado da arte e protocolo de intervenção considerando potenciais fatores causadores de sobrecarga. Universidade Federal do Rio Grande do Norte.

Purdam, C. R., Jonsson, P., Alfredson, H., Lorentzon, R., Cook, J. L. \& Khan, K. M. (2004). A pilot study of the eccentric decline squat in the management of painful chronic patellar tendinopathy. Br J Sports Med, 38, 395-7.

Polit, D., Beck, C., \& Hungler, B. (2004). Fundamentos de pesquisa em enfermagem: métodos, avaliação e utilização (5th ed.). Artmed.

Rutland M, O’Connell D, Brismée J-M, Sizer P, Apte G, O’Connell J. Evidence Supported Rehabilitation of Patellar Tendinopathy. North Am J Sport Phys Ther NAJSPT [Internet]. 2010;5(3):166-78. http://www.pubmedcentral.nih.gov/articlerender.fcgi?artid=2971642\&tool=pmcentrez \&rendertype=abstract

Starkey, C. (2001). Recursos Terapêuticos em Fisioterapia (1ª ed.). Manole.

Spargoli, G. Treatment of rotator cuff tendinopathy as a contractile dysfunction. A Clinical commentary. Phys Ther., Genoa, v. 14, n. 1, p. 148-158, Fev. 2019 Terslev, L., Qvistgaard, E., Torp-Pedersen, S., Laetgaard, J., Danneskiold-Samsøe, B., \& Bliddal, H. (2001). Achados de ultrassom e Power Doppler no joelho de saltador - observações preliminares. European journal of ultrasound , 13 (3), 183-9.

Visnes, H., \& BAHR, R. (2007). A evolução do treinamento excêntrico como tratamento da tendinopatia patelar (joelho de saltador): uma revisão crítica dos programas de exercícios. British Journal of Sports Medicine, 41, 217-223.

Wang, C., Ko, J., Chan, Y., Weng, L., \& Hsu, S. (2007). Extracorporeal shockwave for chronic patellar tendinopathy. The American Journal of Sports Medicine, 35(6), 972-8.

Woodley, B., Newsham-west, R., \& Baxter, G. (2007). Tendinopatia crônica: eficácia do exercício excêntrico. The American Journal of Sports Medicinev.41, p.188-199, 2007, 41, 188-199.

Whittemore, R. (2005). Combinando evidências na pesquisa em enfermagem: métodos e implicações. Pesquisa em enfermagem, 54 (1), 56-62.

Young, M., Cook, J., Purdam, C., Kiss, Z., \& Alfredson, H. (2005). O protocolo de agachamento em declínio excêntrico oferece resultados superiores em 12 meses em comparação com o protocolo excêntrico tradicional para tendinopatia patelar em jogadores de voleibol. British Journal of Sports Medicine, 39 (2), 102-105. 